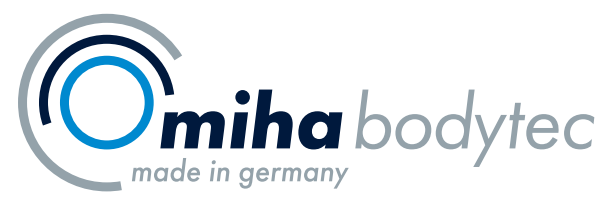

IMPULSE für Prävention und Therapie

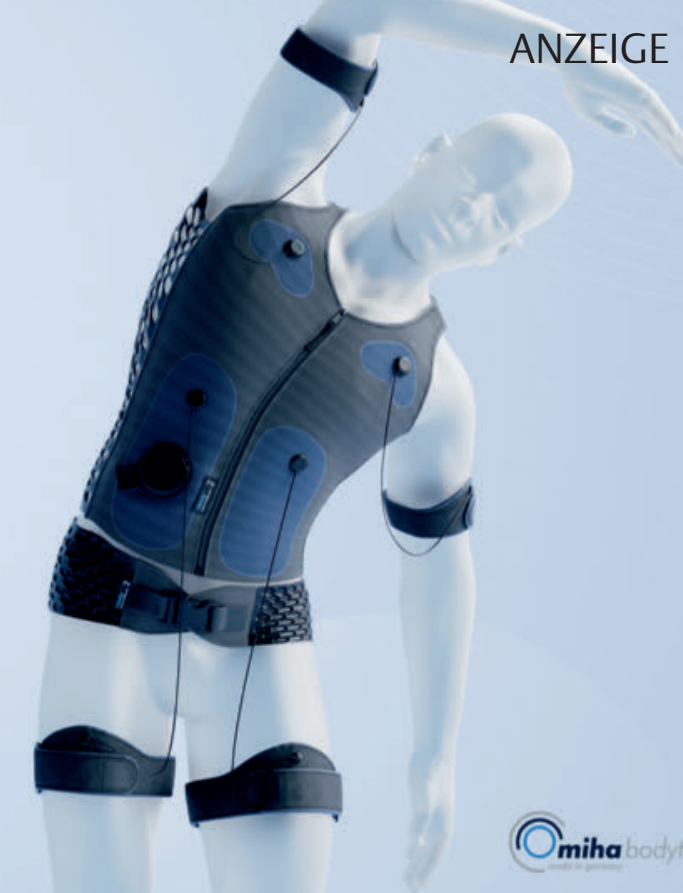

Die Medizinische Elektromyostimulation kombiniert die extern durch niederfrequente Impulse ausgelöste Muskelkontraktion mit willkürlichen Anspannungen. Die isometrischen oder dynamischen Übungen verstärken den Wirkungsgrad der Methode. Durch das simultane Aktivieren von Agonisten, Antagonisten und tiefen Muskelgruppen lässt sich die Muskulatur intensiv, ausdauernd, gelenkschonend und zeitsparend trainieren [7].

\title{
Medizinische EMS bei Rückenschmerzen
}

\author{
Patient*innen mit chronischem, nichtspezifischem Rückenschmerz befinden sich oft in einem \\ Teufelskreis aus Schmerz, Bewegungsangst und -vermeidung [1]. Ohne professionelle Hilfe können \\ sie diesen nur schwer durchbrechen. Hier bietet die Medizinische EMS eine effektive Lösung. Sie ist \\ mindestens so wirksam wie konventionelle Methoden zur Rückenkräftigung und mit 20 Minuten pro \\ Woche deutlich weniger zeitaufwendig.
}

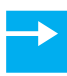

Über drei Viertel aller Deutschen leiden mindestens einmal in ihrem Leben an Rückenschmerzen [3], vorwiegend handelt es sich dabei um lumbale Rückenschmerzen. Die Häufigkeit von Rückenschmerzen steigt mit zunehmendem Alter, wobei Frauen insgesamt etwas häufiger betroffen sind als Männer [4]. In circa 80 Prozent der Fälle liegen nichtspezifische Rückenschmerzen vor [3].

Verschiedene Ursachen begünstigen nichtspezifische Rückenschmerzen. Neben Bewegungsmangel, muskulären Dysbalancen, insuffizienter Rumpfmuskulatur und Fehlbelastungen durch langes Sitzen, einseitige oder schwere körperlicherArbeit können auch psychische Belastungen wie Stress und Sorgen, eine veränderte Schmerzwahrnehmung sowie familiäre Prädispositionen zu den Beschwerden führen $[5,6]$.

Problem der Chronifizierung $\rightarrow$ Weil nichtspezifische Rückenschmerzen häufig chronifizieren [4], klagen etwa zwei Drittel der Betroffenen auch nach zwölf Monaten noch über persistierende
Beschwerden. Die Vermeidung der Chronifizierung ist daher ein wichtiges Ziel der Behandlung nichtspezifischer Rückenschmerzen [4]. Allerdings ist dieses Ziel nicht leicht zu erreichen. Ohne professionelle Unterstützung finden Betroffene nur schwer aus dem Teufelskreis aus Schmerz, Bewegungsangst (Kinesiophobie) und Bewegungsvermeidung wieder heraus [1]. Auch deshalb sind nichtspezifische Rückenschmerzen einer der häufigsten Gründe für die Arbeitsunfähigkeit von Arbeitnehmer*innen und sogar die zweithäufigste Ursache für eine frühzeitige Berentung [4]. Es besteht demnach ein großes Interesse daran, die Chronifizierung von nichtspezifischen Rückenschmerzen zu verhindern und die Therapie so wirkungsvoll und effizient wie möglich zu gestalten.

Deutsche und europäische Leitlinien empfehlen zur Vorbeugung und Therapie nichtspezifischer Rückenschmerzen eine multimodale, individuell auf Patient*innen zugeschnittene Therapie, die neben Physiotherapie, physikalischer Therapie und Psychotherapie auch Beschäftigungstherapie und Edukation enthält [4]. Der erhebliche 
zeitliche Aufwand dieser multimodalen Behandlungsmethode wie auch die häufig bestehende Kinesiophobie sind für viele Betroffene jedoch ein Hemmnis, mit der Therapie zu beginnen [1]. Die Medizinische EMS als zeiteffiziente und innovative Therapieoption bietet daher einen Lösungsansatz mit vielen Vorteilen.

Medizinische EMS nutzt physiologische Muskelkontraktionen $\rightarrow$ Medizinische EMS ist ein nachweislich effektives, zeiteffizientes und gelenkschonendes Behandlungsverfahren [1, 2]. Medizinische EMS verbindet die Wirkung elektrischer Impulse mit willkürlichen Muskelkontraktionen (๑ ABB.). Als Ganzkörper-Anwendung kann Medizinische EMS viele Muskeln gleichzeitig ansprechen. Durch das simultane Aktivieren von Agonisten, Antagonisten und tiefen Muskelgruppen mit niederfrequenten Impulsen kann die Muskulatur intensiv, ausdauernd und gelenkschonend trainiert werden. Und das bei nur 20 Minuten einmal wöchentlich. Die Anwendung findet mittels zertifizierter, antibakterieller Funktionskleidung und Elektrodensystemen unter fachkompetenter, individueller Betreuung (1:1 bzw. max. 1:2) statt. Weil Physiotherapeut*innen aufgrund ihrer Ausbildung befähigt sind, Niederfrequenzgeräte zur elektrischen Muskelstimulation anzuwenden, sind sie für die Durchführung einer Therapie mit Medizinischer EMS prädestiniert. Laut Herstellerangaben bieten bereits über 300 niedergelassene Physiotherapeut*innen die Medizinische EMS als Therapieoption an - mit steigender Tendenz.

Hohe Evidenz für Medizinische EMS $\rightarrow$ Forscher*innen haben die Effekte der Medizinischen EMS im Vergleich zu einem multimodalen Behandlungsprogramm und einem konventionellen Rückentraining untersucht [1,2]. Sie konnten nachweisen, dass die Medizinische EMS eine vergleichbar wirksame, aber deutlich zeiteffektivere Maßnahme zur Prävention und Therapie nichtspezifischer Rückenschmerzen ist.

Konrad et al. 2020 erhoben in einer prospektiven, kontrollierten, nichtrandomisierten klinischen Studie die Wirksamkeit von Medizinischer EMS und multimodaler Behandlung und fanden heraus, dass diese vergleichbar gut wirkt wie die als Goldstandard geltende multimodale Therapie bei der Behandlung von Patient*innen mit nichtspezifischem Rückenschmerz [1]. Während sich die Teilnehmer*innen der Medizinischen EMS-Gruppe einer wöchentlich 20-minütigen Anwendung von Medizinischer EMS über sechs Monate unterzogen, führte die multimodale Gruppe ein 4-wöchiges multimodales Therapieprogramm nach deutschen und europäischen Leitlinien, wie es oben beschrieben wurde, durch. Die Ergebnisse wurden anhand etablierter Fragebögen und Messinstrumente erhoben.

Neben einer klinisch signifikanten Reduktion des Schmerzempfindens (Rückgang um zwei Punkte auf der numerischen Rating-Skala) bei der Medizinischen EMS konnten die Forscher*innen auch eine signifikante Verbesserung der Lebensqualität, des Gesundheitszustandes und der Beeinträchtigung durch das Rückenleiden feststellen [1]. Bei Teilnehmer*innen der multimodalen Gruppe verbesserte sich lediglich die muskuläre Funktion [1]. Patient*innen mit chronischen nichtspezifischen Rückenschmerzen profitieren daher bei einer gleichwertigen Wirksamkeit der beiden Verfahren bei der Medizinischen EMS von einer signifikanten Schmerzreduktion und einer erheblichen Zeitersparnis, womit sich die Medizinische EMS als wirk- same Alternative zum etablierten multimodalen Behandlungsprogramm anbietet.

Eine weitere Studie von Weißenfels et al. aus dem Jahr 2019 konnte belegen, dass die Schmerzintensität bei Patient*innen mit nichtspezifischen Rückenschmerzen vergleichbar signifikant abnimmt wie bei Patient*innen, die ein konventionelles Rückenkräftigungstraining absolvieren [2]. Gleichzeitig nahm die maximale isometrische Muskelkraft in beiden Studiengruppen signifikant zu [2]. Bei dieser Studie verglichen die Forscher*innen die Wirkung einer 12-wöchigen Anwendung von Medizinischer EMS (20 Minuten/Woche) mit einem konventionellen Rückenkräftigungstraining (45 Minuten/Woche), das über 12 Wochen absolviert wurde [2]. Bei vergleichbarer Effizienz überzeugte auch hier die Medizinische EMS aufgrund der erheblichen Zeitersparnis gegenüber dem konventionellen Rückenkräftigungstraining. Insbesondere für Patient*innen mit begrenzten zeitlichen Ressourcen oder Vorbehalten gegenüber konventionellen Trainingsmethoden ist die Medizinische EMS deshalb eine effektive und zeitsparende Methode für den Muskelaufbau, insbesondere für den Bereich der wirbelsäulestabilisierenden Rumpfmuskulatur.

Fazit $\rightarrow$ Bei nichtspezifischen Rückenschmerzen ist Medizinische EMS genauso wirksam, aber wesentlich zeiteffizienter als ein konventionelles Rückenkräftigungstraining oder ein als Goldstandard geltendes multimodales Behandlungsprogramm [1, 2]. Mit einem Zeitaufwand von nur 20 Minuten einmal pro Woche ist Medizinische EMS daher eine effektive und zeitsparende Methode zum Muskelaufbau und zur muskuloskelettalen Stabilisation in Prävention und Therapie nichtspezifischer Rückenschmerzen. N Nach Angaben von miha bodytec

Literaturverzeichnis www.thieme-connect.de/products/physiopraxis > „Ausgabe 1/22“

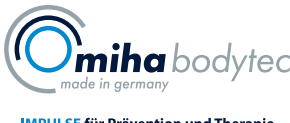

Impressum

Der Artikel erscheint außerhalb des Verantwortungsbereichs der Redaktion sowie der Themenscouts der Zeitschrift physiopraxis.

Eine Sonderpublikation von miha bodytec $\mathrm{GmbH}$, Siemensstr. 1, 86368 Gersthofen

Für Angaben über Dosierungsanweisungen und Applikationsformen kann vom Verlag keine Gewähr übernommen werden. Jeder Benutzerin bzw. jeder Benutzer ist angehalten, durch sorgfältige Prüfung der Beipackzettel der verwendeten Präparate und ggf. nach Konsultation eines Spezialisten festzustellen, ob die dort angegebenen Empfehlungen für Dosierung und die Beachtung von Kontraindikationen gegenüber der Angabe in diesem Artikel abweicht. Eine solche Prüfung ist besonders wichtig bei selten verwendeten Präparaten oder solchen, die neu auf den Markt gebracht worden sind. Jede Dosierung oder Applikation erfolgt auf eigene Gefahr des Benutzenden. 